\title{
Preparation of the tri-arabino di-mycolate fragment of mycobacterial arabinogalactan from defined synthetic mycolic acids
}

Mohammed, Mohsin O.; Baird, Mark; Al-Dulayymi, Juma'a R.

\section{Carbohydrate Research}

DOI:

10.1016/j.carres.2016.11.006

Published: 02/01/2017

Peer reviewed version

Cyswllt i'r cyhoeddiad / Link to publication

Dyfyniad o'r fersiwn a gyhoeddwyd / Citation for published version (APA):

Mohammed, M. O., Baird, M., \& Al-Dulayymi, J. R. (2017). Preparation of the tri-arabino dimycolate fragment of mycobacterial arabinogalactan from defined synthetic mycolic acids. Carbohydrate Research, 437(January), 36-42. https://doi.org/10.1016/j.carres.2016.11.006

\footnotetext{
Hawliau Cyffredinol / General rights

Copyright and moral rights for the publications made accessible in the public portal are retained by the authors and/or other copyright owners and it is a condition of accessing publications that users recognise and abide by the legal requirements associated with these rights.

- Users may download and print one copy of any publication from the public portal for the purpose of private study or research.

- You may not further distribute the material or use it for any profit-making activity or commercial gain

- You may freely distribute the URL identifying the publication in the public portal?
}

Take down policy

If you believe that this document breaches copyright please contact us providing details, and we will remove access to the work immediately and investigate your claim. 


\title{
Preparation of the tri-arabino di-mycolate fragment of mycobacterial arabinogalactan from defined synthetic mycolic acids
}

\author{
Mohsin O. Mohammed ${ }^{1}$, Juma'a R Al Dulayymi and Mark S. Baird* \\ School of Chemistry, Bangor University, Bangor, Wales, LL57 2UW \\ cancer properties [6]. The mechanism of the activity of \\ arabinomycolates is not clear [14].
}

\section{Introduction:}

Tuberculosis (TB) is one of the world's deadliest diseases and is caused by Mycobacterium tuberculosis, which has a single known host (man) [1,2]. The air-borne nature of this bacterium makes the disease highly infectious, and the World Health Organization (WHO) has reported that one third of the world's population is probably infected with latent TB, and that there is a new case every second [3]. The mycobacterial cell wall has a complex structure made up of lipids, glycolipids, polysaccharides and proteins [4]. There has been growing interest in the construction of Darabinooligosaccharides related to mycobacterial cell wall components, arabino-galactan, lipoarabinomannan and arabinomannan from M. tuberculosis [5]. The mycolylarabinogalactan ( $\mathrm{mAG})$ complex is the largest component structure and forms from cross bonding between both Darabinofuranosyl (Araf) and galactofuranosyl (Galf) with a long-chain $\alpha$-alkyl-branched $\beta$-hydroxylated fatty acid, 'mycolic acid' (MA) [6]. The complex lipids and polysaccharides within the cell wall of $M$. tuberculosis are assumed to be the cause of its characteristic pathogenesis

[7].

Anderson and Geiger in 1937 reported the first extraction of arabino-mycolate from the cell wall of Mycobacterium bovis using organic solvent [8]. Azuma et al. reported the isolation of arabinose mycolate under acidic conditions [9]. The structure of the mycolyl-arabinogalactan complex of $M$. tuberculosis was reinterpreted using mass spectrometry and NMR spectroscopy [10,11]. Synthetic arabinofuranosyl oligosaccharides, including a branched pentasaccharide were reported in 1998 [12,13]. In 2005, the preparation of a tetramycolylpentaarabinose (1) (Fig. 1) using a complex natural mixture of MAs was described [14]. However, only in 2010 were structural studies of the composition of the arabinose mycolates of the cell wall of $M$. bovis reported [15]. Ishiwata et al. reported the synthesis of a series of mono- (3), di- and tetra-arabinomycolates (1) found at the terminal position of the cell wall skeleton of BCG from $M$. bovis, using natural MA mixtures extracted from cells. All of the compounds showed strong TNF- $\alpha$ inducing activity in vitro. Such arabinose mycolates have also been reported to show anti-
Although a methoxy trisaccharide of arabinofuranose $\mathbf{2}$ (Fig. 1) has been prepared and used in an acylation reaction with fatty acids such as behenic acid, palmitic acid and butyric acid [16], there are no examples of the synthesis of arabinofuranose oligosaccharides esterified with structurally defined complete synthetic mycolic acids (MAs) which are the main components of the cell wall of $M$. tuberculosis [6]. MAs 4 are $\beta$-hydroxy fatty acids with a long $\alpha$-alkyl side chain (Fig. 2) $[17,18]$.

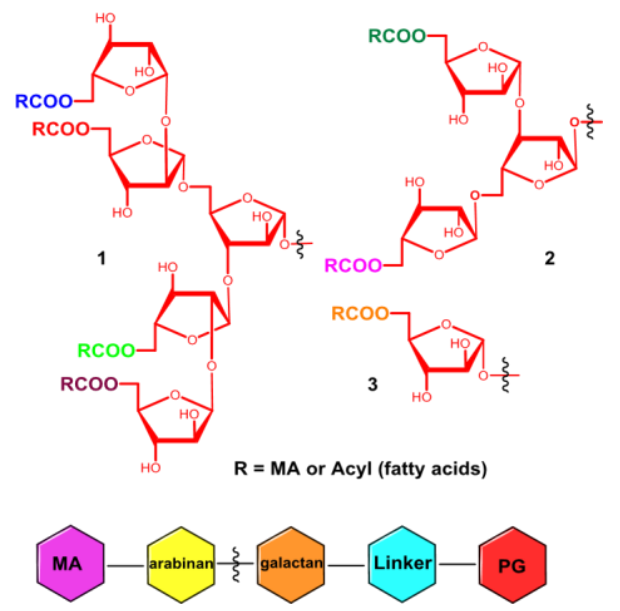

Fig. 1: Structure of targets of mycoloyl-arabinans.

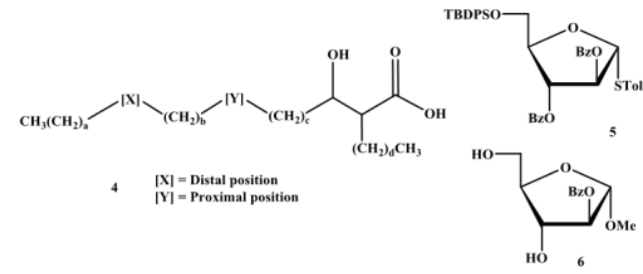

Fig. 2: Generalised MA structure and arabinose building blocks

MAs in M. tuberculosis have 80-90 carbons, compared to those from corynebacterium (30-36 carbons), Rhodococcus (34-38 carbons) and Nocardia (46-60 carbons) [19,20]. Natural mixtures extracted from mycobacteria consist of many individual MA containing a range of functional groups, $\mathrm{X}$ and $\mathrm{Y}$, including cis- and trans-cyclopropanes, cis- and transalkenes, methoxy and keto fragments, and different chain lengths [21].

The aim of this study was to synthesize a series of dimycolated triarabinoses (2), comprising single defined synthetic mycolic acids, in order that the selectivity of their immune stimulatory activity, e.g. in inducing Interleukin 6 (IL-6) and tumor necrosis factor alpha (TNF- $\alpha$ ), could be evaluated. 


\section{Results and Discussion}

\subsection{Synthesis of Trisaccharides}

The target trisaccharide structure $\mathbf{2}$ has two $\alpha$-glycosidic linkages, which can be assembled readily from known building blocks, donor 5 [22] and acceptor 6 [23]. The route to the 2,3$O$-benzoyl-protected thioglycosyl donor $\mathbf{5}$ was slightly modified compared to the literature method (Scheme 1) [22]. D-(-)Arabinose was treated with $\mathrm{HCl}$, freshly prepared by addition of acetyl chloride to anhydrous methanol at $0{ }^{\circ} \mathrm{C}$. Work up with pyridine rather than ammonium carbonate [24], gave methyl- $\alpha$, $\beta$-D-Araf, $7(\mathrm{R}=\mathrm{H})$, with predominant formation of the $\alpha$ anomer $(\alpha / \beta-D, 3: 2)[25,26]$. Rather than esterifying with benzoate, the mixture was directly esterified to give triacetate 7 $(\mathrm{R}=\mathrm{Ac})$ [22]. Condensation of this with $p$-thiotoluene in the presence of boron trifluoride etherate afforded $\mathbf{8}(\mathrm{R}=\mathrm{Ac})$, which on reaction with $\mathrm{NaOCH}_{3}$ in methanol afforded thioglycoside triol $8(\mathrm{R}=\mathrm{H})$. Protection of the primary alcohol as a silyl ether followed by benzoylation of the secondary alcohols gave $\mathbf{5}$.

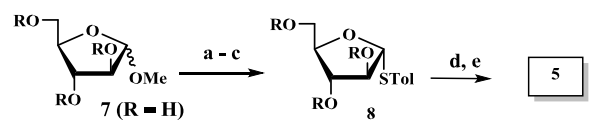

Scheme 1. Reagents and conditions: (a) $\mathrm{Ac}_{2} \mathrm{O}$, pyridine, $0{ }^{\circ} \mathrm{C}, 7(\mathrm{R}=\mathrm{Ac})$ $80 \%$; (b) TolSH (1.2 equiv.), $\mathrm{BF}_{3} . \mathrm{Et}_{2} \mathrm{O}$ (5.1 equiv.), $\mathrm{CH}_{2} \mathrm{Cl}_{2}, 0^{\circ} \mathrm{C} / \mathrm{RT}, 8 \mathrm{~h}, 8$ ( $\mathrm{R}=\mathrm{Ac}), 65 \%$, (c) $\mathrm{NaOCH}_{3}$ (5 equiv.), $\mathrm{CH}_{3} \mathrm{OH}, \mathrm{RT}, 3 \mathrm{~h}, \mathbf{8}(\mathrm{R}=\mathrm{H}), 90 \%$; (d) t- $\mathrm{BuPh}_{2} \mathrm{SiCl}$ (1.5 equiv), imidazole, DMF, $0^{\circ} \mathrm{C} / \mathrm{RT}, 16 \mathrm{~h}, 82 \%$; (e) $\mathrm{BzCl}$ (4.6 equiv.), pyridine, $0^{\circ} \mathrm{C} / \mathrm{RT}, 2 \mathrm{~h}, 83 \%$.

The initial step in the synthetic route to acceptor 6 [23], the separation of the two anomers of $7(\mathrm{R}=\mathrm{H})$, was carried out using two methods (Supplementary data, Schemes 1 and 2).

Liu et al. [16] have reported the coupling of the monosaccharide building blocks to prepare the trisaccharide through the reaction of a 2-O-benzylated glycosyl acceptor and thioglycoside $\mathbf{5}$ using silver trifluoromethane sulfonate and Niodosuccinimide in dry $\mathrm{CH}_{2} \mathrm{Cl}_{2}$. In the present work, the benzoylated glycosyl acceptor $\mathbf{6}$ and thioglycoside 5 were reacted under the reported conditions (Scheme 2). Trisaccharide 9a was obtained as a single stereoisomer in $87 \%$ yield, which is similar to that reported for the benzyl compound [16]. The product showed the expected three acetal signals in the proton NMR spectrum, two broad singlets at $\delta$

5.10 and 5.33 , for the newly formed $\alpha$-glycosidic links, and a third singlet at 5.56; these correlated by HSQC with three signals in the carbon spectrum for the glycosidic links at $\delta 107.0,106.0$ and 105.3 respectively. Compound 9a was deprotected with sodium methoxide to give $\mathbf{9 b}$ as a thick oil in $81 \%$ yield. This was benzylated to protect the five secondary hydroxyl groups using benzyl bromide and sodium hydride in dry DMF to give 9c. Desilylation of the two primary hydroxyl groups gave 10a. Direct esterification of the primary hydroxyl group of Araf with a carboxyl group in natural mycolic acid mixtures has been achieved in a low yield (30\%) [14], while activating the sugar as a tosylate raised the yield to $79 \%$. Therefore, the two hydroxyl groups in compound 10a were tosylated using $p$-toluenesulfonyl chloride in dry pyridine in the presence of catalytic 4dimethylaminopyridine to afford the tosylate 10b (Scheme 2).

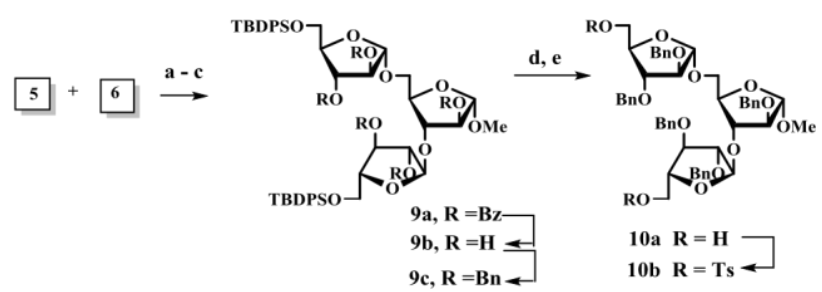

Scheme 2. Reagents and conditions: (a) NIS, AgOTf, (9a, 87\%); (b) $\mathrm{NaOCH}_{3}$ (cat.), $\mathrm{CH}_{3} \mathrm{OH}$, rt, 2 h, $(9 \mathbf{b}, 86 \%)$; (C) NaH, BnBr, DMF, 0 ${ }^{\circ} \mathrm{C} / \mathrm{rt} 16$ h, (9c, 79\%); (d) TBAF, THF, $0^{\circ} \mathrm{C} / \mathrm{rt}, 16 \mathrm{~h}$ (10a 77\%); (e) TsCl, pyridine, DMAP, $\mathrm{CH}_{2} \mathrm{Cl}_{2}$ at $0{ }^{\circ} \mathrm{C}(\mathbf{1 0 b}, 74 \%)$.

The next step was the coupling of trisaccharide $\mathbf{1 0 b}$ with the structurally defined synthetic mycolic acids, which had been prepared earlier [27-29]. Firstly, a model tri-arabinodi mycolate was prepared by coupling tosylate 10b with palmitic acid through the alkylative esterification strategy using cesium hydrogen carbonate in dry DMF: THF at 70 ${ }^{\circ} \mathrm{C}$ for four days [14], to give compound 11a, which was then debenzylated to give compound 12a (Scheme 3). The synthesis of this glycolipid has been reported using a different method. Data obtained for 12a was identical to that reported [16].

A series of triarabino-dimycolates were then prepared according to the above procedure; the structures of the mycolic acid moieties, including $\alpha$-, methoxy- and ketoclasses are shown in Scheme 3. In each case, the products the proton NMR spectra included the expected three characteristic broad single-hydrogen singlets for the acetal hydrogens in the region $\delta 4.9-5.2$.
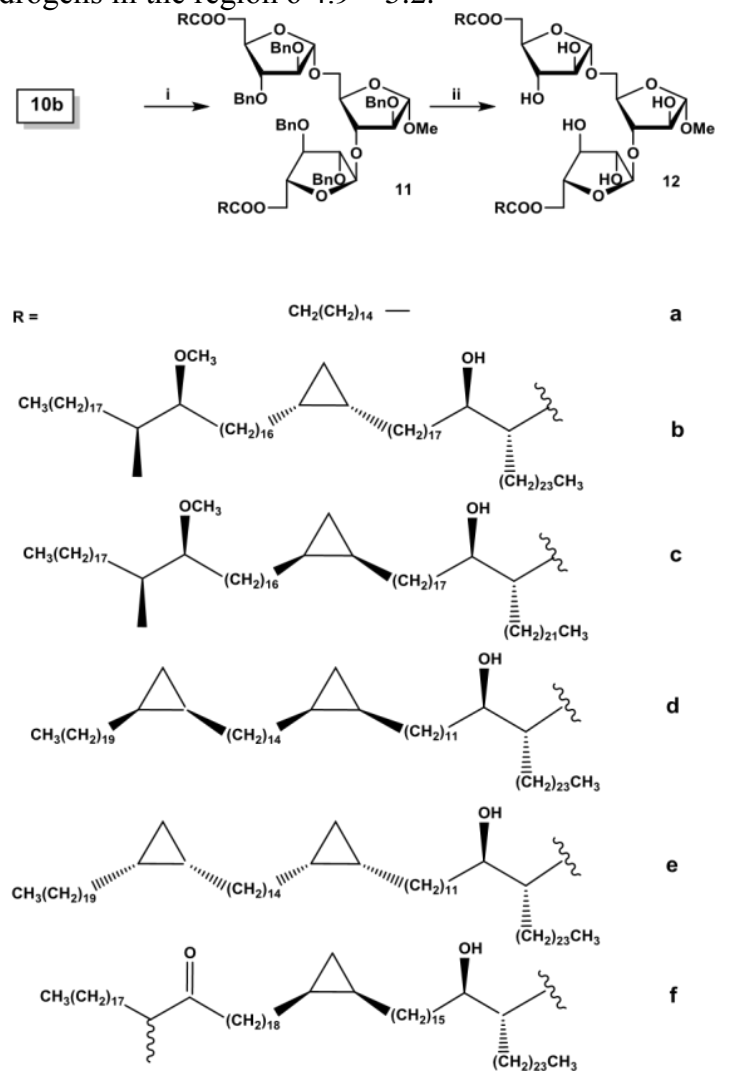

Scheme 3. Reagents and conditions: (i) $\mathrm{CsHCO}_{3}$, THF-DMF, 4 days, $70^{\circ} \mathrm{C}$; (ii) $\mathrm{Pd}(\mathrm{OH})_{2}, \mathrm{H}_{2}, \mathrm{CH}_{2} \mathrm{Cl}_{2}-\mathrm{MeOH}$, RT, overnight. 


\subsection{IL-6 and TNF- $\alpha$ secretion assays with the synthesized compounds}

Ishiwata et al. reported the synthesis of a series of mono-, diand tetra-arabinomycolates found in the terminal position of the cell-wall skeleton of bacillus calmette Guerin from $M$. bovis, by using natural mycolic acid mixtures extracted from the cell wall and showed they differentially induce TNF- $\alpha$ secretion in a murine macrophage cell line, some at levels similar to trehalose dimycolate (TDM) [14]. Arabinomycolates obtained by acid hydrolysis from the originally prepared CWS (SMP-105) of $M$. bovis BCG Tokyo 172, consisting mainly of mono-arabinose mono-mycolate, pentaarabinose tetra-mycolate and hexaarabinose tetra-mycolate fractions, significantly induced TNF- $\alpha$ production with an intensity comparable to that of cell-wall skeleton, a potent adjuvant, and enhanced delayed type per sensitivity reactions against inactivated tumour cells. The induced TNF- $\alpha$ production was completely dependent on TLR2 and MyD88 pathways [6]. To provide an initial evaluation of the activities of synthesised tri-arabino di-mycolates reported here (12c and 12f) as immune potentiators, secretion inducing assays for IL6 and TNF- $\alpha$, cytokines involved in inflammation, were conducted, in comparison with TDM and synthetic monoarabino mono-mycolates [30]. Surprisingly, neither compound was strongly stimulatory in either assay when compared to synthetic TDMs, trehalose monomycolates or arabinose mycolates [31, 32]. Further work to evaluate the biological effects of these molecules is under way.

\section{Experimental Section}

For general experimental detail, see Supplementary Information. Unless otherwise stated, all products were single components by TLC and by proton and carbon NMR.

3.1: $\quad$ Methyl 2,3-di-O-benzyl-5-O-p-toluenesulfonyl- $\alpha$ Darabinofuranosyl-( $1 \rightarrow 3)$-[2,3-di-O-benzyl-5-O-p-toluenesulfonyl- $\alpha$-D-arabinofuranosyl- $(1 \rightarrow 5)]-2-O$-benzyl- $\alpha-D$ - Araf 10b

(a) Molecular sieves $4 \AA$ (6.6 g) were added to a stirred solution of the acceptor $6(0.9 \mathrm{~g}, 3.3 \mathrm{mmol})$ and the donor $5(6.0 \mathrm{~g}, 8.5 \mathrm{mmol})$ in dry $\mathrm{CH}_{2} \mathrm{Cl}_{2}(30 \mathrm{~mL})$ at r.t. under a nitrogen atmosphere. The reaction mixture was stirred for $30 \mathrm{~min}$. then cooled to $-60{ }^{\circ} \mathrm{C}$ and $\mathrm{N}$-iodosuccinimide $(2.09 \mathrm{~g}, 9.29 \mathrm{mmol})$ was added followed by the addition of AgOTf (0.36 g, $1.40 \mathrm{mmol})$. The mixture was stirred at the same temperature until the color became red/dark brown and TLC showed no starting material, then it was quenched by the addition of triethylamine $(1 \mathrm{~mL})$ until it turned yellow. The mixture was diluted with $\mathrm{CH}_{2} \mathrm{Cl}_{2}(100 \mathrm{~mL})$ and filtered through celite and the solvent was evaporated under reduced pressure. Column chromatography on silica eluting with hexane/ethyl acetate (5:1) gave compound 9a as a thick oil $(4.2 \mathrm{~g}, 87 \%)$ [Found (MALDI) $(\mathrm{M}+\mathrm{Na})^{+}:$1447.6, $\mathrm{C}_{83} \mathrm{H}_{84} \mathrm{NaO}_{18} \mathrm{Si}_{2}$, requires: 1447.5$],[\mathrm{C}]^{20}{ }_{D}+16(c$ $\left.0.1, \mathrm{CHCl}_{3}\right)$ which showed $\delta_{\mathrm{H}}\left(400 \mathrm{MHz}, \mathrm{CDCl}_{3}\right)$ :

$8.07(2 \mathrm{H}, \mathrm{d}, J 7.9 \mathrm{~Hz}), 8.02-7.91(8 \mathrm{H}, \mathrm{m}), 7.70-7.62(9 \mathrm{H}, \mathrm{m})$, $7.59-7.41(8 \mathrm{H}, \mathrm{m}), 7.40-7.28(18 \mathrm{H}, \mathrm{m}), 5.64(1 \mathrm{H}, \mathrm{d}, J 4.5 \mathrm{~Hz})$, $5.61(1 \mathrm{H}, \mathrm{d}, J 4.2 \mathrm{~Hz}), 5.56(1 \mathrm{H}, \mathrm{s}), 5.57(1 \mathrm{H}, \mathrm{s}), 5.53(1 \mathrm{H}, \mathrm{br} . \mathrm{s})$,
$5.41(1 \mathrm{H}, \mathrm{s}), 5.32(1 \mathrm{H}$, br. s), $5.10(1 \mathrm{H}$, br. s), $4.49-4.35(4 \mathrm{H}$, $\mathrm{m}), 4.08(1 \mathrm{H}, \mathrm{dd}, J 11.3,5.1 \mathrm{~Hz}), 3.97(5 \mathrm{H}, \mathrm{m}), 3.44(3 \mathrm{H}, \mathrm{s})$, $1.01(9 \mathrm{H}, \mathrm{s}), 0.98(9 \mathrm{H}, \mathrm{s}) ; \delta_{\mathrm{C}}\left(101 \mathrm{MHz}, \mathrm{CDCl}_{3}\right): 165.5,165.4$, 165.2 ,

$165.1,135.63,135.6,135.5,133.3,133.2,133.15,133.1,133.0$ $129.96,129.9,129.8,129.78,129.7,129.6,129.4,129.3,129.2$, 129.1, 128.4, 128.31, 128.3, 128.2, 127.6, 107.0, 106.0, 105.3 84.0, 83.4, 82.5, 82.0, 81.8, 81.6, 80.6, 77.2, 77.1, 66.1, 63.5, 63.3 ,

54.7, 26.7, 26.6, 19.3, 19.2; $v_{\max }: 3069,3010,2929,2859,1724$, $1602,1451,1070,708 \mathrm{~cm}^{-1}$.

(b) A solution of sodium methoxide (2 mL, $1 \mathrm{M}$, in methanol) was added to a stirred solution of $9 \mathrm{a}(0.20 \mathrm{~g}, 0.14$ mmol) in dry $\mathrm{MeOH}: \mathrm{CH}_{2} \mathrm{Cl}_{2}(1: 1,5 \mathrm{~mL})$ at r.t. until a $\mathrm{pH}$ of 11 was obtained. The mixture was stirred at r.t. for $2 \mathrm{~h}$ then the solvent was evaporated under reduced pressure to give an oil. The residue was purified by column chromatography on silica eluting with $\mathrm{CHCl}_{3}: \mathrm{MeOH}(5: 2)$ to give $9 \mathbf{b}$ compound as a thick oil $(0.1 \mathrm{~g}, 83 \%)$ [Found

(MALDI) $(\mathrm{M}+\mathrm{Na})^{+}:$927.1, $\mathrm{C}_{48} \mathrm{H}_{64} \mathrm{NaO}_{13} \mathrm{Si}_{2}$, requires: 927.3], [ $\left.\square\right]$ ${ }^{22}{ }_{D}+50\left(c 0.10, \mathrm{CHCl}_{3}\right)$ which showed $\delta_{\mathrm{H}}\left(400 \mathrm{MHz}, \mathrm{CDCl}_{3}+\right.$ few drops $\left.\mathrm{CD}_{3} \mathrm{OD}\right): 7.49-7.34(20 \mathrm{H}, \mathrm{m}), 5.19(1 \mathrm{H}, \mathrm{s}), 5.14(1 \mathrm{H}, \mathrm{s})$, $4.86(1 \mathrm{H}, \mathrm{s}), 4.25-4.20(2 \mathrm{H}, \mathrm{m}), 4.18(1 \mathrm{H}$, br. d, $J 1.8 \mathrm{~Hz}), 4.12$ (2H, br. d, J 3.8 Hz), $4.05-4.02(4 \mathrm{H}, \mathrm{m}), 4.01-3.95(2 \mathrm{H}, \mathrm{m}), 3.80$ $(1 \mathrm{H}, \mathrm{dd}, J 11.4,2.3 \mathrm{~Hz}), 3.77-3.73(2 \mathrm{H}, \mathrm{m}), 3.70(2 \mathrm{H}$, br. d, $J 11.3$ $\mathrm{Hz}), 3.34(3 \mathrm{H}, \mathrm{s}), 3.01-2.70(4 \mathrm{H}, \mathrm{m}), 1.10-0.99(18 \mathrm{H}, \mathrm{m}) ; \delta_{\mathrm{C}}$ (101 MHz, $\mathrm{CDCl}_{3}+$ few drops $\left.\mathrm{CD}_{3} \mathrm{OD}\right): 135.7,135.6,135.5$, $129.5,128.3,128.0,127.9,127.8,109.1,108.6,108.4$ 88.0, 87.4, 83.8, 82.4, 79.3, 78.9, 78.4, 77.8, 77.7, 66.1, 64.0, $63.85,54.8,26.7,26.6$; $v_{\max }: 3445,3010,2928,2860,1494$, $1050,824 \mathrm{~cm}^{-1}$.

(c) Compound 9b (0.2 g, $0.2 \mathrm{mmol})$ in dry DMF:THF(1:1) ( $4 \mathrm{~mL}$ ) was added dropwise (1 drop each 10 second) to a stirred suspension of $\mathrm{NaH}(0.1 \mathrm{~g}, 8.3 \mathrm{mmol})(60 \% \mathrm{w} / \mathrm{w}$, dispersion in mineral oil, washed with petrol three times) at r.t. under nitrogen. The mixture was stirred for $30 \mathrm{~min}$ then benzyl bromide $(0.1 \mathrm{~mL}, 0.8 \mathrm{mmol})$ in dry DMF $(1 \mathrm{~mL})$ was added, stirred at r.t. for $16 \mathrm{~h}$, then quenched by slow addition of methanol $(3 \mathrm{~mL})$, and water $(10 \mathrm{~mL})$. The organic layer was separated and the aqueous layer was re-extracted with ethyl acetate $(2 \times 50 \mathrm{~mL})$. The combined organic layers were washed with water $(20 \mathrm{~mL})$ and brine $(20 \mathrm{~mL})$, dried and the solvent evaporated under reduced pressure. Column chromatography on silica eluting with petrol/ethyl acetate $(5: 1)$ gave the title compound as a thick oil $(0.23 \mathrm{~g}, 77 \%)$ [Found (MALDI) $(\mathrm{M}+\mathrm{Na})^{+}:$1377.0, $\mathrm{C}_{83} \mathrm{H}_{94} \mathrm{NaO}_{13} \mathrm{Si}_{2}$, requires: 1377.6], [] ${ }^{22}{ }_{D}$ $+40\left(c 0.1, \mathrm{CHCl}_{3}\right)$ which showed $\delta_{\mathrm{H}}\left(400 \mathrm{MHz}, \mathrm{CDCl}_{3}\right): 7.66$ $-7.51(7 \mathrm{H}, \mathrm{m}), 7.42-7.17(38 \mathrm{H}, \mathrm{m}), 5.19(1 \mathrm{H}, \mathrm{s})$,

$5.17(1 \mathrm{H}, \mathrm{s}), 4.94(1 \mathrm{H}, \mathrm{s}), 4.59-4.37(10 \mathrm{H}, \mathrm{m}), 4.28(1 \mathrm{H}, \mathrm{dd}$, $J$ 6.3, $2.3 \mathrm{~Hz}), 4.22-4.14(2 \mathrm{H}, \mathrm{m}), 4.14-4.03(5 \mathrm{H}, \mathrm{m}), 4.03$ $-3.95$

(2H, m), $3.86-3.74(5 \mathrm{H}, \mathrm{m}), 3.37(3 \mathrm{H}, \mathrm{s}), 1.03(18 \mathrm{H}, \mathrm{s}) ; \delta \mathrm{c}$ $(101$

$\left.\mathrm{MHz}, \mathrm{CDCl}_{3}\right)$ : 135.7, 135.65, 135.62, 135.6, 129.6, 129.54, $129.51,128.5,128.4,128.35,128.34,128.3,128.22,128.2$, $127.9,127.8,127.79,127.75,127.73,127.66,127.63,127.6$, 127.56, 127.53, 127.4, 127.35, 127.33, 127.3, 127.28, 107.2,

(b) Palladium (II) hydroxide on activated charcoal (20\% $\mathrm{Pd}(\mathrm{OH})_{2}-\mathrm{C}, 12 \mathrm{mg}, 0.3$ fold by weight) was added to a stirred 
$106.5,105.4,88.5,88.3,88.0,83.1,82.8,82.3,81.8,81.2,80.4$, $72.0,71.9,71.73,71.7,71.6,66.2,63.5,63.3,54.7,26.8,26.7$; $v_{\max }$ : $3466,3050,3017,2958,2930,2858,1493,1112,701 \mathrm{~cm}^{-1}$.

(d) Tetrabutylammonium fluoride $(0.3 \mathrm{~mL}, 0.3 \mathrm{mmol}, 1 \mathrm{M})$ was added dropwise to a stirred solution of $9 \mathrm{c}(0.2 \mathrm{~g}, 0.147 \mathrm{mmol})$ in dry THF $(10 \mathrm{~mL})$ at $0{ }^{\circ} \mathrm{C}$ under nitrogen atmosphere. The mixture was allowed to reach r.t. and stirred for $16 \mathrm{~h}$ then the solvent was evaporated under reduced pressure to give an oil which was purified by column chromatography on silica eluting with hexane/ethyl acetate $(1: 1)$ to give compound $\mathbf{1 0 a}$ as a colourless thick oil $(0.1 \mathrm{~g}$, $77 \%$ ) [Found (MALDI) $(\mathrm{M}+\mathrm{Na})^{+}:$901.3, $\mathrm{C}_{51} \mathrm{H}_{58} \mathrm{NaO}_{13}$, requires : 901.3], [] ${ }^{20}{ }_{D}+90\left(c 0.1, \mathrm{CHCl}_{3}\right)$, which showed $\delta_{\mathrm{H}}(400 \mathrm{MHz}$, $\left.\mathrm{CDCl}_{3}\right): 7.38-7.21(25 \mathrm{H}, \mathrm{m}), 5.16(1 \mathrm{H}, \mathrm{s}), 5.12(1 \mathrm{H}, \mathrm{d}, J 1.2 \mathrm{~Hz})$, $4.96(1 \mathrm{H}, \mathrm{d}, J 1.2 \mathrm{~Hz}), 4.61-4.42(9 \mathrm{H}, \mathrm{m}), 4.38(1 \mathrm{H}, \mathrm{d}, J 12.0 \mathrm{~Hz})$, $4.30(1 \mathrm{H}, \mathrm{dd}, J 7.4,3.7 \mathrm{~Hz}), 4.25(1 \mathrm{H}$, ddd, $J 6.3,5.2,2.9 \mathrm{~Hz}), 4.16$ $-4.07(3 \mathrm{H}, \mathrm{m}), 4.03(1 \mathrm{H}, \mathrm{dd}, J 3.7,1.2 \mathrm{~Hz}), 4.01(1 \mathrm{H}, \mathrm{dd}, J 3.7,1.2$ $\mathrm{Hz}), 3.95(1 \mathrm{H}, \mathrm{dd}, J 11.6,3.8 \mathrm{~Hz}), 3.91(1 \mathrm{H}, \mathrm{dd}, J 6.6,2.9 \mathrm{~Hz}), 3.85$ $-3.71(4 \mathrm{H}, \mathrm{m}), 3.66(1 \mathrm{H}, \mathrm{dd}, J 12.2,5.2 \mathrm{~Hz}), 3.59(1 \mathrm{H}, \mathrm{dd}, J 12.2$, $5.8 \mathrm{~Hz}), 3.40(3 \mathrm{H}, \mathrm{s}), 2.84$ (1H, br. s), 2.48 (1H, br. s); $\delta_{\mathrm{C}}(101 \mathrm{MHz}$, $\left.\mathrm{CDCl}_{3}\right): 137.7$,

137.6, 137.4, 137.3, 137.2, 128.5, 128.44, 128.43, 128.4, 128.38, 127.96, 127.94, 127.9, 127.87, 127.83, 127.8, 127.71, 106.9, 106.2, $105.8,88.7,88.2,87.5,83.0,82.9,82.3,81.9,80.9,79.7,72.3,72.2$, $72.0,71.9,71.8,64.9,62.8,62.7,54.9$; $v_{\max }: 3467,3050,3017$, 2926, 2861, 1609, 1494, 1050, $824 \mathrm{~cm}^{-1}$.

(e) 4-Toluenesulfonyl chloride (2.82 g, $14.7 \mathrm{mmol})$ was added to a stirred solution of $\mathbf{1 0 a}(1.30 \mathrm{~g}, 1.47 \mathrm{mmol})$, pyridine $(1.17 \mathrm{~g}, 1.19 \mathrm{~mL}, 14.7 \mathrm{mmol})$ and DMAP (catalytic amount) in dry

$\mathrm{CH}_{2} \mathrm{Cl}_{2}(10 \mathrm{~mL})$ at $0{ }^{\circ} \mathrm{C}$ under nitrogen. The mixture was allowed to reach r.t. and stirred for $16 \mathrm{~h}$, then diluted with ethyl acetate $(100 \mathrm{~mL})$ and water $(50 \mathrm{~mL})$, the organic layer was separated and the aqueous layer was re-extracted with ethyl acetate $(3 \times 50 \mathrm{~mL})$. The combined organic layers were washed with water $(50 \mathrm{~mL})$ and brine $(50 \mathrm{~mL})$, dried. The solvent was evaporated under reduced pressure to give an oil; column chromatography on silica eluting with hexane/ethyl acetate

(1:1) afforded the title compound as a colorless thick oil 10b

$(1.3 \mathrm{~g}, \quad 74 \%)$ [Found (MALDI) $(\mathrm{M}+\mathrm{Na})^{+}:$1209.2,

$\mathrm{C}_{65} \mathrm{H}_{70} \mathrm{NaO}_{17} \mathrm{~S}_{2}$, requires: 1209.3], [0] ${ }^{22}{ }_{D}+71\left(c 0.10, \mathrm{CHCl}_{3}\right)$ which

showed $\delta_{\mathrm{H}}\left(400 \mathrm{MHz}, \mathrm{CDCl}_{3}\right): 7.7(2 \mathrm{H}, \mathrm{d}, J 8.3 \mathrm{~Hz})$,

$7.8(2 \mathrm{H}, \mathrm{d}, J 8.4 \mathrm{~Hz}), 7.37-7.23(29 \mathrm{H}, \mathrm{m}), 5.09$ (1H, br. s), 5.08 (1H, br. s), $4.93(1 \mathrm{H}, \mathrm{s}), 4.61-4.42(9 \mathrm{H}, \mathrm{m}), 4.35(1 \mathrm{H}, \mathrm{d}, J 12.0$ $\mathrm{Hz}), 4.30-4.24(1 \mathrm{H}, \mathrm{m}), 4.22-4.10(6 \mathrm{H}, \mathrm{m}), 4.10-4.05(2 \mathrm{H}$, $\mathrm{m}), 4.0(1 \mathrm{H}, \mathrm{dd}, J 3.3,1.2 \mathrm{~Hz}), 3.96(1 \mathrm{H}, \mathrm{dd}, J 3.1$,

$1.0 \mathrm{~Hz}), 3.87(2 \mathrm{H}, \mathrm{dd}, J 6.1,3.1 \mathrm{~Hz}), 3.84(1 \mathrm{H}, \mathrm{dd}, J 12.0,4.0$ $\mathrm{Hz}), 3.69$ (1H, dd, J 12.0, $2.5 \mathrm{~Hz}), 3.38(3 \mathrm{H}, \mathrm{s}), 2.38(3 \mathrm{H}, \mathrm{s}), 2.4$ $(3 \mathrm{H}, \mathrm{s}) ; \delta_{\mathrm{C}}\left(101 \mathrm{MHz}, \mathrm{CDCl}_{3}\right): 144.8,144.7,137.5,137.4,137.3$, 137.2, 137.1, 132.7, 132.6, 129.8, 129.7, 128.4, 128.38, 128.35, $128.33,128.3,128.0,127.93,127.9,127.85,127.82,127.8$, $127.7,127.69,127.67,106.9,106.4,105.5,88.0,87.6$, $87.4,82.8,82.7,80.5,80.4,79.0,78.7,72.2,72.0,71.8,71.81$, $71.7,68.7,68.6,65.7,54.8,21.5 ; v_{\max }: 3088,3064,3031,2924$, $2862,1598,1454,1177,738 \mathrm{~cm}^{-1}$.

3.2: Methyl5-O-[(2R)-2-(1-hydroxy-18-[(1S,2R)-2-[(17S, 18S) 17methoxy-18-methylhexatriacontyl) cyclopropyl]octadecyl]hexacosanoate $)]-\alpha-D$-arabinofuranosyl- $(1 \rightarrow 3)-5-O-[(2 R)-2$ (1hydroxy-18-[(1S,2R)-2-[(17S,18S)-17-methoxy-18- methylhexatriacontyl)cyclopropyl]octadecyl]hexacosanoate)]$\alpha$-D-arabinofuranosyl- $(1 \rightarrow 5)]-\alpha-D$-Araf $\mathbf{1 2 b}$

(a) Cesium hydrogencarbonate $(0.065 \mathrm{~g}, 0.335 \mathrm{mmol})$ was added to a stirred solution of $\mathbf{1 0 b}(0.040 \mathrm{~g}, 0.033 \mathrm{mmol})$ and (2R)-2-\{1-hydroxy-18-[(1S,2R)-2-[(17S,18S)-17-

methoxy18-

methylhexatriacontyl]cyclopropyl]octadecyl \}hexacosanoic acid [28] (0.092 g, $0.073 \mathrm{mmol})$ in dry DMF : THF (1:5, 3 $\mathrm{mL})$ at r.t. under a nitrogen atmosphere. The mixture was stirred at $70{ }^{\circ} \mathrm{C}$ for 4 days, then worked up and purified as before to afford 11b as a colourless thick oil $(0.051 \mathrm{~g}, 45 \%)$ [Found (MALDI) $(\mathrm{M}+\mathrm{Na})^{+}: \quad 3371.9, \quad \mathrm{C}_{221} \mathrm{H}_{390} \mathrm{NaO}_{19}$, requires: 3371.9], [व] ${ }^{23}{ }_{D}+35\left(c 0.1, \mathrm{CHCl}_{3}\right)$ which showed $\delta_{\mathrm{H}}\left(400 \mathrm{MHz}, \mathrm{CDCl}_{3}\right): 7.37-7.23(25 \mathrm{H}, \mathrm{m}), 5.18(1 \mathrm{H}$, br. s), $5.13(1 \mathrm{H}, \mathrm{br}$.

s), $4.91(1 \mathrm{H}$, br. s), $4.49-4.37(9 \mathrm{H}, \mathrm{m}), 4.34(1 \mathrm{H}, \mathrm{d}, J 11.7$ $\mathrm{Hz}), 4.32-4.24(6 \mathrm{H}, \mathrm{m}), 4.18(1 \mathrm{H}$, ddd, $J 9.7,6.3,3.0 \mathrm{~Hz})$, $4.11(1 \mathrm{H}, \mathrm{dd}, J 4.4,2.7 \mathrm{~Hz}), 4.08(1 \mathrm{H}$, br. d, $J 2.9 \mathrm{~Hz}), 4.00$ $(1 \mathrm{H}, \mathrm{dd}, J 2.9,0.6 \mathrm{~Hz}), 3.96(1 \mathrm{H}$, br. d, $J 2.3 \mathrm{~Hz}), 3.93(1 \mathrm{H}$, dd, $J 7.8,3.9 \mathrm{~Hz}), 3.88-3.78(2 \mathrm{H}, \mathrm{m}), 3.76(1 \mathrm{H}, \mathrm{dd}, J 11.4$, $1.8 \mathrm{~Hz}), 3.67-3.57(2 \mathrm{H}, \mathrm{m}), 3.37(3 \mathrm{H}, \mathrm{s}), 3.35(6 \mathrm{H}, \mathrm{s}), 2.99$ $-2.93(2 \mathrm{H}, \mathrm{m}), 2.67(1 \mathrm{H}, \mathrm{d}, J 6.1 \mathrm{~Hz}), 2.65(1 \mathrm{H}, \mathrm{d}, J 6.5 \mathrm{~Hz})$, $2.41(2 \mathrm{H}, \mathrm{dt}, J 9.0,5.8 \mathrm{~Hz}), 1.68-1.03(294 \mathrm{H}, \mathrm{m}), 0.89$ $(12 \mathrm{H}, \mathrm{t}, J 6.8 \mathrm{~Hz}), 0.86(6 \mathrm{H}, \mathrm{d}, J 6.9 \mathrm{~Hz}), 0.71-0.61(4 \mathrm{H}$, $\mathrm{m}), 0.57(2 \mathrm{H}, \mathrm{dt}, J 7.6,4.0 \mathrm{~Hz}),-0.33(2 \mathrm{H}$, br. q, $J 5.1 \mathrm{~Hz})$; $\delta_{\mathrm{C}}\left(101 \mathrm{MHz}, \mathrm{CDCl}_{3}\right): 175.1,175.0,137.7,137.6,137.5$, $137.4,137.2$, 128.5, 128.41, 128.4, 127.95, 127.9, 127.8, $127.73,127.7,107.0,106.3,105.5,88.2,87.9,87.8,85.4$, 83.7, 80.6, 80.4,

79.3, 79.1, 72.3, 72.2, 72.1, 71.98, 71.8, 65.6, 63.0, 57.7, 54.8, $51.9,51.7,35.3,32.4,31.9,30.5,30.2,29.97,29.9,29.7$, 29.66, 29.5, 29.4, 28.7, 27.6, 26.2, 22.7, 15.8, 14.9, 14.1, 10.9 ; $v_{\max }: 3517,3063,3031,2922,2852,1736,1466,1101$, 757 cmsolution of compound $11 b(0.042 \mathrm{~g}, 0.014 \mathrm{mmol})$ in dry $\mathrm{CH}_{2} \mathrm{Cl}_{2}: \mathrm{MeOH}(1: 1,2 \mathrm{~mL})$ at r.t. under a hydrogen. The mixture was stirred for $16 \mathrm{~h}$, then filtered and the solvent was evaporated under reduced pressure; column chromatography on silica eluting with $\mathrm{CHCl}_{3}: \mathrm{MeOH}(5: 1)$ gave the title compound 12b as a thick oil $(0.03 \mathrm{~g}, 82 \%)$ [Found $(\mathrm{M}+\mathrm{Na})^{+}$: 2921.8, $\mathrm{C}_{186} \mathrm{H}_{360} \mathrm{NaO}_{19}$, requires: 2921.7], [व] ${ }^{20}{ }_{D}+21$ (c 0.10,

$\left.\mathrm{CHCl}_{3}\right)$ which showed $\delta_{\mathrm{H}}\left(500 \mathrm{MHz}, \mathrm{CDCl}_{3}+\right.$ few drops $\mathrm{CD}_{3} \mathrm{OD}$ ): 4.98 (1H, br. s), 4.95 (1H, br. s), 4.77 (1H, br. s), 4.40 (1H, dd, $J 11.8,4.3 \mathrm{~Hz}), 4.33(1 \mathrm{H}, \mathrm{dd}, J 11.6,4.5 \mathrm{~Hz})$, 4.24 (1H, dd, $J 11.6,4.7 \mathrm{~Hz}), 4.15(1 \mathrm{H}, \mathrm{dd}, J 11.6,4.3 \mathrm{~Hz})$, $4.13-4.07(2 \mathrm{H}, \mathrm{m}), 4.07-3.94(5 \mathrm{H}, \mathrm{m}), 3.94-3.80(3 \mathrm{H}$, $\mathrm{m}), 3.67-3.55(3 \mathrm{H}, \mathrm{m}), 3.36(3 \mathrm{H}, \mathrm{s}), 3.32(6 \mathrm{H}, \mathrm{s}), 2.96-$ $2.90(2 \mathrm{H}, \mathrm{m}), 2.37-2.30(2 \mathrm{H}, \mathrm{m}), 1.61-0.97(301 \mathrm{H}, \mathrm{m})$, $0.82(12 \mathrm{H}, \mathrm{t}, J 7.0 \mathrm{~Hz}), 0.79(6 \mathrm{H}, \mathrm{d}, J 6.9 \mathrm{~Hz}), 0.64-0.55$ $(4 \mathrm{H}, \mathrm{m})$,

$0.50(2 \mathrm{H}, \mathrm{dt}, J 8.0,4.0 \mathrm{~Hz}),-0.39(2 \mathrm{H}$, br. q, $J 5.1 \mathrm{~Hz}) ; \delta_{\mathrm{C}}$ (126 MHz, $\mathrm{CDCl}_{3}+$ few drops $\left.\mathrm{CD}_{3} \mathrm{OD}\right): 175.1,175.0$, 109.1, 107.9, 107.2, 85.5, 83.0, 82.4, 81.8, 81.0, 79.8, 77.9, $72.5,72.45$,

$65.9,63.4,63.1,57.5,54.8,52.8,50.0,35.2,34.8,34.7$, 32.2

$31.8,30.3,30.1,29.82,29.8,29.62,29.6,29.56,29.5,29.4$, $29.3,29.2,29.1,28.6,27.4,27.3,25.96,25.2,25.2,22.5$, 15.6 
$14.7,13.9,10.7 ; v_{\max }: 3401,2919,2851,1733,1467,1099,720$ $\mathrm{cm}^{-1}$.

\section{3: Methyl 5-O-(2-[(R)-1-hydroxy-18-[(1R,2S)-2-[(17S, 18S)-17-methoxy-18- \\ methylhexatriacontyl]cyclopropyl]octadecyl]-tetracosanoate)- $\alpha$-D-arabino-furanosyl- $(1 \rightarrow 3)[5-O-(2-[(R)-1$-hydroxy-18- $[(1 R, 2 S)-2-[(17 S, 18 S)-17$ methoxy-18-methyl- \\ hexatriacontyl]cyclopropyl]octadecyl]tetracosanoate)- $\alpha-D$ - arabinofuranos-yl- $(1 \rightarrow 5)]-\alpha-D$-Araf \\ 12c}

(a) Cesium hydrogencarbonate $(0.081 \mathrm{~g}, 0.417 \mathrm{mmol})$ was added to a stirred solution of $\mathbf{1 0 b}(0.050 \mathrm{~g}, 0.042 \mathrm{mmol})$ and $(2 R)-2-\{(1 R)-1$-hydroxy-18-[(2S)-2-[(17S,18S)-17-methoxy18ethylhexatriacontyl]cyclopropyl]octadecyl \}tetracosanoic acid [33] $(0.113 \mathrm{~g}, 0.092 \mathrm{mmol})$ in dry DMF : THF (1:5, $6 \mathrm{~mL})$ at r.t. under nitrogen. The mixture was stirred at $70{ }^{\circ} \mathrm{C}$ for 4 days, then work up and purification as before gave 11c as a colourless thick oil (50 mg, 36\%) [Found (M+Na) ${ }^{+}: 3315.8808, \mathrm{C}_{217} \mathrm{H}_{382} \mathrm{NaO}_{19}$, requires: 3315.8818$],[0]^{23}+40(c$

$\left.0.1, \mathrm{CHCl}_{3}\right)$ which showed $\delta_{\mathrm{H}}\left(400 \mathrm{MHz}, \mathrm{CDCl}_{3}\right): 7.39-7.22$ $(25 \mathrm{H}, \mathrm{m}), 5.18(1 \mathrm{H}, \mathrm{s}), 5.13(1 \mathrm{H}, \mathrm{s}), 4.91(1 \mathrm{H}, \mathrm{s}), 4.61-4.42$ $(9 \mathrm{H}, \mathrm{m}), 4.38(1 \mathrm{H}, \mathrm{d}, J 12.0 \mathrm{~Hz}), 4.30-4.23(6 \mathrm{H}, \mathrm{m}), 4.22-$ $4.16(1 \mathrm{H}, \mathrm{m}), 4.14-4.05(2 \mathrm{H}, \mathrm{m}), 4.03(1 \mathrm{H}, \mathrm{dd}, J 3.2,1.0 \mathrm{~Hz})$, $3.98(1 \mathrm{H}, \mathrm{dd}, J 3.2,1.0 \mathrm{~Hz}), 3.95(1 \mathrm{H}, \mathrm{dd}, J 12.0,4.6 \mathrm{~Hz}), 3.86$ - $3.80(2 \mathrm{H}, \mathrm{m}), 3.78(1 \mathrm{H}, \mathrm{dd}, J 12.0,2.3 \mathrm{~Hz}), 3.67-3.57(2 \mathrm{H}$, m), $3.37(3 \mathrm{H}, \mathrm{s}), 3.35(6 \mathrm{H}, \mathrm{s}), 3.01-2.92(2 \mathrm{H}, \mathrm{m}), 2.67(2 \mathrm{H}, \mathrm{br}$. s), $2.45-2.37(2 \mathrm{H}, \mathrm{m}), 1.72-1.07(285 \mathrm{H}, \mathrm{m}), 0.89(12 \mathrm{H}, \mathrm{t}, J$ $6.8 \mathrm{~Hz}), 0.86(6 \mathrm{H}, \mathrm{d}, J 6.9 \mathrm{~Hz}), 0.71-0.60(4 \mathrm{H}, \mathrm{m}), 0.61$ $-0.53(2 \mathrm{H}, \mathrm{dt}, J 7.6,4.0 \mathrm{~Hz}),-0.32(2 \mathrm{H}$, br. q, J $5.2 \mathrm{~Hz}) ; \delta_{\mathrm{C}}$ $\left(101 \mathrm{MHz}, \mathrm{CDCl}_{3}\right)$ : 175.1, 174.9, 137.6, 137.51, 137.5, 137.4, $137.2,128.45,128.41,128.4,128.38,128.37,127.9,127.85$, $127.8,127.74,127.7,107.0,106.3,105.5,88.2,87.9,87.8$, 85.4, 83.7, 83.6, 80.6, 80.4, 79.3, 79.1, 72.3, 72.2, 72.1, 72.0, 71.9, 71.7, 65.5, 63.0, 57.7, 54.8, 51.8, 51.7, 35.3, 32.4, 31.9, $30.5,30.2,29.98,29.9,29.7,29.6,29.5,29.4,28.7,27.6,27.4$, $26.2,22.7,15.7,14.8,14.1,10.9 ; v_{\max }: 3436,2920,2851,1733$, $1734,1174,734 \mathrm{~cm}^{-1}$.

(b) Palladium (II) hydroxide on activated charcoal (20\% $\mathrm{Pd}(\mathrm{OH})_{2}-\mathrm{C}, 0.01 \mathrm{~g}, 0.30$ fold by weight) was added to a stirred solution of compound $11 \mathrm{c}(0.037 \mathrm{~g}, 0.010 \mathrm{mmol})$ in dry $\mathrm{CH}_{2} \mathrm{Cl}_{2}$ : $\mathrm{MeOH}(1: 1,2 \mathrm{~mL})$ at r.t. under hydrogen. After $16 \mathrm{~h}$, work up and purification as before gave the title compound $12 \mathrm{c}$ as a white oil (21 mg, 65\%) [Found (M+Na) ${ }^{+}: 2865.6424, \mathrm{C}_{182} \mathrm{H}_{352} \mathrm{NaO}_{19}$, requires: 2865.6470$],[0]{ }_{D}^{16}+24\left(c 0.1, \mathrm{CHCl}_{3}\right)$ which showed $\delta_{\mathrm{H}}\left(400 \mathrm{MHz}, \mathrm{CDCl}_{3}+\right.$ few drops $\left.\mathrm{CD}_{3} \mathrm{OD}\right)$ :

$5.02(1 \mathrm{H}, \mathrm{d}, J 1.5 \mathrm{~Hz}), 4.98(1 \mathrm{H}$, br. s), $4.80(1 \mathrm{H}$, br. s), $4.46(1 \mathrm{H}$, dd, $J 11.7,4.3 \mathrm{~Hz}), 4.37(1 \mathrm{H}, \mathrm{dd}, J 11.6,4.8 \mathrm{~Hz}), 4.27(1 \mathrm{H}, \mathrm{dd}$, $J$ 11.7, $4.9 \mathrm{~Hz}), 4.16(1 \mathrm{H}, \mathrm{dd}, J 12.0,4.8 \mathrm{~Hz}), 4.14-4.08(2 \mathrm{H}$, $\mathrm{m}), 4.08-3.99(5 \mathrm{H}, \mathrm{m}), 3.95-3.87(3 \mathrm{H}, \mathrm{m}), 3.62$ $-3.52(3 \mathrm{H}, \mathrm{m}), 3.35(3 \mathrm{H}, \mathrm{s}), 3.31(6 \mathrm{H}, \mathrm{s}), 2.94-2.85(2 \mathrm{H}, \mathrm{m})$, $2.44-2.34(2 \mathrm{H}, \mathrm{m}), 1.37-1.13(292 \mathrm{H}, \mathrm{m}), 0.85(12 \mathrm{H}, \mathrm{t}, J 6.8 \mathrm{~Hz})$, $0.82(6 \mathrm{H}, \mathrm{d}, J 6.9 \mathrm{~Hz}), 0.66-0.57(4 \mathrm{H}, \mathrm{m}), 0.56-0.49$ $(2 \mathrm{H}, \mathrm{dt}, J 7.6,4.0 \mathrm{~Hz}),-0.37(2 \mathrm{H}$, br. q, J $5.2 \mathrm{~Hz}) ; \delta_{\mathrm{C}}(101 \mathrm{MHz}$, $\mathrm{CDCl}_{3}+$ few drops $\left.\mathrm{CD}_{3} \mathrm{OD}\right): 175.2,175.0,109.1,107.9,107.2$, $85.5,82.9,82.5,82.0,81.8,81.0,80.9,79.7,78.0,72.5$, $66.9,65.9,63.1,57.6,54.8,52.8,35.2,32.3,31.8,30.4,30.1$, $29.8,29.7,29.66,29.6,29.5,29.3,29.2,28.6,27.4,26.0,22.5$, $15.7,14.7,14.0,10.8 ; v_{\max }: 3412,2919,2850,1731,1466,1099$, $720 \mathrm{~cm}^{-1}$.
3.4: Methyl 5-O-(2-[(R)-1-hydroxy-12-\{(1R,2S)-2-(14-[(1R, $2 S)$-2-eicosylcyclopropyl]tetradecyl]cyclopropyl)dodecyl)hexacosanoate $)-\alpha-D$-arabinofuranosyl- $(1 \rightarrow 3)-[5-O-(2-$ $[(R) 1-h y d r o x y-12-\{(1 R, 2 S)-2-(14-[(1 R, 2 S)-2$-eicosylcyclopropyl] tetradecyl]cyclopropyl $\}$ dodecyl)hexacosanoate )- $\alpha$-Darabinofuranosyl- $(1 \rightarrow 5)]-\alpha-D$-Araf $\mathbf{1 2 d}$

(a) Cesium hydrogencarbonate $(0.089 \mathrm{~g}, 0.458 \mathrm{mmol})$ was added to a stirred solution of $\mathbf{1 0 b}(0.055 \mathrm{~g}, 0.046 \mathrm{mmol})$ and $\quad(2 R)-2-\{(1 R)-1$-hydroxy-12-[(1R)-2-[14-[(2S)-2eicosylcyclopropyl]tetradecyl]cyclopropyl]dodecyl $\}$ hexaco sanoic acid [27] (0.115 g, $0.101 \mathrm{mmol})$ in dry DMF : THF $(1: 5,6 \mathrm{~mL})$ at r.t. under hydrogen. The mixture was stirred at $70{ }^{\circ} \mathrm{C}$ for 4 days then work up and purification as before afforded 11d as a colourless oil (56 $\mathrm{mg}, 18 \%$ ) [Found $(\mathrm{M}+\mathrm{Na})^{+}: 3139.7057, \mathrm{C}_{207} \mathrm{H}_{358} \mathrm{NaO}_{17}$, requires: 3139.7041$]$, [व] ${ }^{23}{ }_{D}+72(c$

$\left.0.1, \mathrm{CHCl}_{3}\right)$ which showed $\delta_{\mathrm{H}}\left(400 \mathrm{MHz}, \mathrm{CDCl}_{3}\right): 7.37-7.22$ $(25 \mathrm{H}, \mathrm{m}), 5.18$ (1H, br. s), 5.14 (1H, br. s), 4.92 (1H, br. s), $4.61-4.43(9 \mathrm{H}, \mathrm{m}), 4.35(1 \mathrm{H}, \mathrm{d}, J 12.0 \mathrm{~Hz}), 4.32-4.25(6 \mathrm{H}$, $\mathrm{m}), 4.22-4.16(1 \mathrm{H}, \mathrm{m}), 4.09-4.03(2 \mathrm{H}, \mathrm{m}), 4.01(1 \mathrm{H}, \mathrm{dd}, J$ 3.0, $1.0 \mathrm{~Hz}), 3.97(1 \mathrm{H}, \mathrm{dd}, J 3.0,1.0 \mathrm{~Hz}), 3.92(1 \mathrm{H}, \mathrm{dd}, J 12.0$, $7.5 \mathrm{~Hz}), 3.88-3.80(2 \mathrm{H}, \mathrm{m}), 3.77(1 \mathrm{H}, \mathrm{dd}, J 12.0,2.2 \mathrm{~Hz})$, $3.62-3.55$ (2H, m), $3.38(3 \mathrm{H}, \mathrm{s}), 2.68-2.52$ (2H, m), $2.46-$ $2.36(2 \mathrm{H}, \mathrm{m}), 1.70-1.06(268 \mathrm{H}, \mathrm{m}), 0.89(12 \mathrm{H}, \mathrm{t}, J 6.8 \mathrm{~Hz})$, $0.71-0.62(8 \mathrm{H}, \mathrm{m}), 0.60-0.54(4 \mathrm{H}, \mathrm{dt}, J 7.6,4.0 \mathrm{~Hz}),-0.32$ $(4 \mathrm{H}$, br. q, $J 5.2 \mathrm{~Hz}) ; \delta_{\mathrm{C}}\left(101 \mathrm{MHz}, \mathrm{CDCl}_{3}\right): 175.1,174.9$, $137.6,137.51,137.5,137.4,137.2,128.5,128.4,128.38$, $128.37,128.0,127.9,127.8,127.71,127.7,107.0,106.3$, $105.5,88.2,87.9,87.8,83.6,80.6,80.4,79.3,79.1,72.3$, 72.2 ,

$72.0,71.9,71.7,65.6,63.0,54.8,51.85,51.7,35.4,35.2,31.9$, $30.2,29.8,29.75,29.7,29.6,29.5,29.4,29.2,28.7,27.5,27.4$, 25.7, 22.7, 18.5, 18.3, 15.7, 14.1, 10.9; $v_{\max }: 3584,3064,3032$, 2918, 2850, 1733, 1455, 1018, $732 \mathrm{~cm}^{-1}$.

(b) Palladium (II) hydroxide on activated charcoal ( $20 \% \mathrm{Pd}(\mathrm{OH})_{2}-\mathrm{C}, 6.6 \mathrm{mg}, 0.30$ fold by weight) was added to a stirred solution of compound $11 \mathrm{~d}(0.030 \mathrm{~g}, 0.009 \mathrm{mmol})$ in dry $\mathrm{CH}_{2} \mathrm{Cl}_{2}: \mathrm{MeOH}(1: 1,2 \mathrm{~mL})$ at r.t. under hydrogen. After $16 \mathrm{~h}$, work up and purification as before gave the title compound 12d as a colourless oil (17 mg, 64\%) [Found $(\mathrm{M}+\mathrm{Na})^{+}:$2689.4696, $\mathrm{C}_{172} \mathrm{H}_{328} \mathrm{NaO}_{17}$, requires: 2689.4694], $[\mathrm{C}]{ }_{D}^{16}+36\left(c 0.1, \mathrm{CHCl}_{3}\right)$ which showed $\delta_{\mathrm{H}}(400 \mathrm{MHz}$, $\mathrm{CDCl}_{3}+$ few drops $\left.\mathrm{CD}_{3} \mathrm{OD}\right): 5.03(1 \mathrm{H}, \mathrm{d}, J 1.5 \mathrm{~Hz}), 4.99$ (1H, br. s), $4.81(1 \mathrm{H}$, br.

s), $4.48(1 \mathrm{H}, \mathrm{dd}, J 11.9,4.3 \mathrm{~Hz}), 4.39(1 \mathrm{H}, \mathrm{dd}, J 11.7,4.7$ $\mathrm{Hz}), 4.28$ (1H, dd, $J 11.5,4.8 \mathrm{~Hz}), 4.17(1 \mathrm{H}, \mathrm{dd}, J 11.4,4.3$ $\mathrm{Hz}), 4.13-3.09$ (2H, m), $4.06-3.98$ (5H, m), $3.97-3.86$ $(3 \mathrm{H}, \mathrm{m})$,

$3.69-3.58(3 \mathrm{H}, \mathrm{m}), 3.36(3 \mathrm{H}, \mathrm{s}), 2.51-2.42(2 \mathrm{H}, \mathrm{m}), 2.42$

$2.35(2 \mathrm{H}, \mathrm{m}), 1.70-1.00(271 \mathrm{H}, \mathrm{m}), 0.86(12 \mathrm{H}, \mathrm{t}, J 6.8 \mathrm{~Hz})$, $0.67-0.58(8 \mathrm{H}, \mathrm{m}), 0.57-0.50(4 \mathrm{H}, \mathrm{dt}, J 7.6,4.0 \mathrm{~Hz})$, 0.36

(4H, br. q, $J 5.1 \mathrm{~Hz})$; $\delta_{\mathrm{C}}\left(101 \mathrm{MHz}, \mathrm{CDCl}_{3}+\right.$ few drops $\left.\mathrm{CD}_{3} \mathrm{OD}\right)$ : 175.2, 175.0, 109.0, 107.8, 107.3, 83.1, 82.9, 81.5 ,

81.1, 80.0, 77.97, 72.99, 72.9, 65.7, 63.6, 63.2, 54.9, 52.8, $52.7,31.9,30.3,30.2,29.8,29.75,29.7,29.6,29.5,29.4$, 28.7, 
27.4, 22.7, 15.8, 14.1, 10.9; $v_{\max }$ : v. br. 3400, 2919, 2851, 1732, $1467,1099,760 \mathrm{~cm}^{-1}$.

\section{5: $\quad$ Methyl-5-O-(2-\{(R)-1-hydroxy-12-[(1S,2R)-2-(14-[(1S, $2 R)-2$}

eicosylcyclopropyl]tetradecyl)cyclopropyl]dodecyl $\}$ hexacosan oate)- $\alpha$-D-arabinofuran-osyl- $(1 \rightarrow 3)-[5-O-(2-\{(R) 1$-hydroxy-

$12-[(1 S, 2 R)-2-(14-[(1 S, 2 R)-2-i c o s y l c y c l o p r o p-y l]$ tetradecyl $)$ cyclopropyl]dodecyllhexacosanoate)- $\alpha$-Darabinofuranosyl$(1 \rightarrow 5)]-\alpha-D$-Araf $12 \mathrm{e}$

(a) Cesium hydrogencarbonate $(0.081 \mathrm{~g}, 0.417 \mathrm{mmol})$ was added to a stirred solution $10 \mathrm{~b}(0.050 \mathrm{~g}, 0.042 \mathrm{mmol})$ and $2\{(R)$ 1-hydroxy-12-[(1S,2R)-2-(14-[(1S,2R)-2-

eicosylcyclopropyl]tetradecyl)cyclopropyl]dodecyl \}hexacosan oic acid [27] (0.105 g, $0.092 \mathrm{mmol})$ in dry DMF : THF (1:5, 3 $\mathrm{mL}$ ) at r.t. under hydrogen. The mixture was stirred at $70{ }^{\circ} \mathrm{C}$ for 4 days, then work and purification as before gave a colourless oil, compound 11e (31 mg, 11\%) [Found (MALDI) $(\mathrm{M}+\mathrm{Na})^{+}$: 3139.7, $\mathrm{C}_{207} \mathrm{H}_{358} \mathrm{NaO}_{17}$ requires: 3139.7], [व] ${ }^{23}{ }_{D}+80$ (c 0.1,

$\left.\mathrm{CHCl}_{3}\right)$ which showed $\delta_{\mathrm{H}}\left(400 \mathrm{MHz}, \mathrm{CDCl}_{3}\right): 7.35-7.27(25 \mathrm{H}$, $\mathrm{m}), 5.18(1 \mathrm{H}$, br. s), 5.13 (1H, br. s), $4.91(1 \mathrm{H}$, br. s), $4.61-4.39$ (9H, m), $4.40(1 \mathrm{H}, \mathrm{d}, J 12.8 \mathrm{~Hz}), 4.35$ (1H, dd, $J$ 9.4, $4.6 \mathrm{~Hz}), 4.32-4.23(5 \mathrm{H}, \mathrm{m}), 4.21-4.17$ (1H, m), $4.11-$ $4.05(2 \mathrm{H}, \mathrm{m}), 4.00(1 \mathrm{H}$, br. d, J $2.7 \mathrm{~Hz}), 3.98-3.95(1 \mathrm{H}, \mathrm{m})$, $3.95-3.91(1 \mathrm{H}, \mathrm{m}), 3.90-3.85(2 \mathrm{H}, \mathrm{m}), 3.76(1 \mathrm{H}, \mathrm{dd}, J 11.7$, $2.2 \mathrm{~Hz}), 3.66-3.57(2 \mathrm{H}, \mathrm{m}), 3.37(3 \mathrm{H}, \mathrm{s}), 2.69-2.62(2 \mathrm{H}, \mathrm{m})$, $2.45-2.36(2 \mathrm{H}, \mathrm{m}), 1.64-1.07(268 \mathrm{H}, \mathrm{m}), 0.89(12 \mathrm{H}, \mathrm{t}, J 6.9$ $\mathrm{Hz}), 0.70-0.61(8 \mathrm{H}, \mathrm{m}), 0.56(4 \mathrm{H}, \mathrm{dt}, J 7.6,4.0 \mathrm{~Hz})$, -

$0.33(4 \mathrm{H}$, br. q, $J 5.2 \mathrm{~Hz}) ; \delta_{\mathrm{C}}\left(101 \mathrm{MHz}, \mathrm{CDCl}_{3}\right): 175.1,175.0$, 137.7, 137.5, 137.4, 137.3, 137.2, 128.5, 128.4, 128.38, 128.37, $127.95,127.9,127.8,127.73,127.7,107.0,106.3,105.5,88.2$, 87.9, 87.8, 83.6, 80.6, 79.2, 79.1, 72.3, 72.2, 72.1,

72.0, 71.9, 71.7, 65.6, 63.0, 60.4, 54.8, 51.8, 51.7, 31.9, 30.2, $29.72,29.7,29.6,29.5,29.4,28.7,22.6,15.8,14.1,10.9$; $v_{\max }$ : $3524,3035,2918,2850,1735,1466,1018,734 \mathrm{~cm}^{-1}$.

(b) Palladium (II) hydroxide on activated charcoal $(20 \%$ $\mathrm{Pd}(\mathrm{OH})_{2}-\mathrm{C}, 8.2 \mathrm{mg}, 0.30$ fold by weight) was added to a stirred solution of compound $11 \mathrm{e}(0.027 \mathrm{~g}, 0.008 \mathrm{mmol})$ in dry $\mathrm{CH}_{2} \mathrm{Cl}_{2}$ : $\mathrm{MeOH}(1: 1,2 \mathrm{~mL})$ at r.t. under hydrogen. After $16 \mathrm{~h}$, work up and purification as before gave the title compound 12e as a colourless oil $(23.1 \mathrm{mg}, 97 \%)$ [Found (MALDI) $(\mathrm{M}+\mathrm{Na})^{+}$: 2689.4, $\mathrm{C}_{172} \mathrm{H}_{328} \mathrm{NaO}_{17}$, requires: 2689.4], [व] ${ }^{20}{ }_{D}+40$

(c $0.1, \mathrm{CHCl}_{3}$ ) which showed $\delta_{\mathrm{H}}\left(400 \mathrm{MHz}, \mathrm{CDCl}_{3}+\right.$ few drops $\left.\mathrm{CD}_{3} \mathrm{OD}\right): 4.94$ (1H, d, $\left.J 1.3 \mathrm{~Hz}\right), 4.92$ (1H, br. s), 4.73 (1H, br. s), $4.35(1 \mathrm{H}, \mathrm{dd}, J 11.8,4.7 \mathrm{~Hz}), 4.29(1 \mathrm{H}, \mathrm{dd}, J 11.5,3.9 \mathrm{~Hz})$, $4.20(1 \mathrm{H}, \mathrm{dd}, J 11.5,4.8 \mathrm{~Hz}), 4.13(1 \mathrm{H}, \mathrm{dd}, J 11.7,4.8 \mathrm{~Hz})$, $4.09-4.02(2 \mathrm{H}, \mathrm{m}), 4.02-3.92(5 \mathrm{H}, \mathrm{m}), 3.89-3.77(3 \mathrm{H}, \mathrm{m})$, $3.61-3.49$ (3H, m), $3.28(3 \mathrm{H}, \mathrm{s}), 2.38-2.29(2 \mathrm{H}, \mathrm{m}), 1.51-$ $0.94(274 \mathrm{H}, \mathrm{m}), 0.79(12 \mathrm{H}, \mathrm{t}, J 6.8 \mathrm{~Hz}), 0.61-0.52(8 \mathrm{H}, \mathrm{m})$, $0.47(4 \mathrm{H}, \mathrm{dt}, J 7.6,4.0 \mathrm{~Hz}),-0.42(4 \mathrm{H}$, br. q, $J 5.1 \mathrm{~Hz}) ; \delta_{\mathrm{C}}(101$ $\mathrm{MHz}, \mathrm{CDCl}_{3}+$ few drops $\left.\mathrm{CD}_{3} \mathrm{OD}\right): 175.1,175.0,109.0,107.9$, 107.3, 82.9, 82.2, 81.8, 81.6, 81.0, 80.9, 79.7, 77.8, 77.5, 72.3, 66.0, 63.4, 63.1, 54.7, 52.7, 49.6, 34.7, 31.7, 30.0, 29.9, 29.6, 29.5, 29.4, 29.2, 29.1, 29.0, 28.5, 27.2, 22.4, 15.5, 13.8, 10.7, $10.6 ; v_{\max }: 3400,2918,2850,1735,1467,1008 \mathrm{~cm}^{-1}$.

3.6: Methyl5-O-\{2-((1R)-1-hydroxy-16-[(1R,2S)-2-(20methyl19-oxo-octatriacontyl)cyclopropyl] hexadecyl)hexacosanoyl $\}-\alpha$ $D$-arabinofuranosyl-( $1 \rightarrow 3)-[2,3-d i-O-b e n z y l-5 O-\{2-(1 R)-1$ hydroxy-16-[(1R,2S)-2-(20-methyl-19- oxooctatriacontyl)cyclopropyl]hexadecyl)hexacosanoyl $\}-\alpha-$ Darabinofuranosyl- $(1 \rightarrow 5)]-\alpha-D$-Araf $\mathbf{1 2 f}$

(a) Cesium hydrogencarbonate $(0.081 \mathrm{~g}, 0.417 \mathrm{mmol})$ was added to a stirred solution of $\mathbf{1 0 b}(0.050 \mathrm{~g}, 0.042 \mathrm{mmol})$ and (2R)-2-\{(1R)-1-hydroxy-16-[(2S)-2-[20-methyl-19-oxooctatriacontyl]cyclopropyl]hexadecyl $\}$ hexacosanoic acid [29] $(0.114 \mathrm{~g}, 0.092 \mathrm{mmol})$ in dry DMF : THF (1:5, $6 \mathrm{~mL})$ at r.t. under nitrogen. The mixture was stirred at $70{ }^{\circ} \mathrm{C}$ for 4 days, then work up and purification as before gave compound 11 as a colourless thick oil $(0.05 \mathrm{~g}, 36 \%)$ [Found (MALDI)

$(\mathrm{M}+\mathrm{Na})^{+}:$3339.7, $\mathrm{C}_{219} \mathrm{H}_{382} \mathrm{NaO}_{19}$, requires: 3339.8], [प] ${ }^{23}{ }_{D}+62$ ( $\left.c 0.1, \mathrm{CHCl}_{3}\right)$ which showed $\delta_{\mathrm{H}}\left(400 \mathrm{MHz}, \mathrm{CDCl}_{3}\right)$ : $7.34-7.25$ $(25 \mathrm{H}, \mathrm{m}), 5.18(1 \mathrm{H}$, br. s), $5.13(1 \mathrm{H}$, br. s), $4.91(1 \mathrm{H}, \mathrm{br}$. s), $4.60-4.40(9 \mathrm{H}, \mathrm{m}), 4.34(1 \mathrm{H}, \mathrm{d}, J 11.8 \mathrm{~Hz}), 4.32-4.24$ $(4 \mathrm{H}, \mathrm{m}), 4.18(1 \mathrm{H}, \mathrm{dt}, J 10.7,3.5 \mathrm{~Hz}), 4.11(1 \mathrm{H}, \mathrm{dd}, J 4.1$, $2.9 \mathrm{~Hz}), 4.08(1 \mathrm{H}$, br. d, J 3.0 Hz), $4.04-4.01(1 \mathrm{H}, \mathrm{m}), 4.00$ (1H, br. d, J 2.4 Hz), $3.96-3.94(2 \mathrm{H}, \mathrm{m}), 3.93(1 \mathrm{H}, \mathrm{dd}, J$ 7.4, $4.4 \mathrm{~Hz}), 3.88-3.80(2 \mathrm{H}, \mathrm{m}), 3.76(1 \mathrm{H}, \mathrm{dd}, J 12.0,2.0 \mathrm{~Hz})$, $3.70-3.55(3 \mathrm{H}, \mathrm{m}), 3.37(3 \mathrm{H}, \mathrm{s}), 2.67(1 \mathrm{H}$, br. s), $2.55-$ $2.34(8 \mathrm{H}, \mathrm{m}), 1.69-1.10(288 \mathrm{H}, \mathrm{m}), 1.06(6 \mathrm{H}, \mathrm{d}, J 6.9 \mathrm{~Hz})$, $0.89(12 \mathrm{H}, \mathrm{t}, J 6.8), 0.66-0.62(4 \mathrm{H}, \mathrm{m}), 0.61-0.51(2 \mathrm{H}, \mathrm{dt}$, $J$ 7.6, $4.0 \mathrm{~Hz}),-0.32(2 \mathrm{H}$, br. q, $J 5.1 \mathrm{~Hz}) ; \delta_{\mathrm{C}}(101 \mathrm{MHz}$, $\left.\mathrm{CDCl}_{3}\right): 215.2,175.1,174.96,137.7,137.5,137.4,137.2$, $128.4,128.41,128.38,128.36,128.0,127.9,127.8,127.73$, $127.7,107.0,106.3,105.5,88.2,87.9,87.85,83.7,80.6$, $80.4,79.3,79.1$,

72.3, 72.2, 72.1, 71.97, 71.8, 68.9, 65.6, 63.0, 54.8, 51.8, 51.7, $50.9,46.3,41.1,33.0,32.1,31.9,30.2,29.7,29.66,29.6,29.5$, $29.49,29.4,29.36,29.3,29.2,28.7,27.4,27.3,25.7,23.7$, $22.8,22.7,16.4,15.7,14.1,10.9$; $v_{\max }: 3522,3066,2919,2851$, $1735,1712,1467,1111,756,720 \mathrm{~cm}^{-1}$.

(b) Palladium (II) hydroxide on activated charcoal $(20 \%$ $\mathrm{Pd}(\mathrm{OH})_{2}-\mathrm{C}, 13.8 \mathrm{mg}, 0.30$ fold by weight) was added to a stirred solution of compound $\mathbf{1 1 f}(0.046 \mathrm{~g}, 0.013 \mathrm{mmol})$ in dry $\mathrm{CH}_{2} \mathrm{Cl}_{2}: \mathrm{MeOH}(1: 1,2 \mathrm{~mL})$ at r.t. under hydrogen. After $16 \mathrm{~h}$, work up and chromatography as before gave the title compound $\mathbf{1 2 f}$ as a colourless oil (26 mg, 65\%) [Found $(\mathrm{M}+\mathrm{Na})^{+}:$2889.6404, $\mathrm{C}_{184} \mathrm{H}_{352} \mathrm{NaO}_{19}$, requires: 2889.6470], []$^{23}{ }_{D}+33\left(c 0.1, \mathrm{CHCl}_{3}\right)$ which showed $\delta_{\mathrm{H}}(400 \mathrm{MHz}$, $\mathrm{CDCl}_{3}+$ few drops $\left.\mathrm{CD}_{3} \mathrm{OD}\right): 5.00(1 \mathrm{H}, \mathrm{d}, J 1.8 \mathrm{~Hz}), 4.96$ (1H, d, $J 0.6 \mathrm{~Hz}), 4.79$ (1H, br. s), 4.43 (1H, dd, $J 12.2,4.6$ $\mathrm{Hz}), 4.35$ (1H, dd, $J 12.2,4.9 \mathrm{~Hz}), 4.26(1 \mathrm{H}, \mathrm{dd}, J 11.8,5.0$ $\mathrm{Hz}), 4.16$ (1H, dd, J 11.4, 4.2 Hz), $4.13-4.07(2 \mathrm{H}, \mathrm{m}), 4.04$ - $3.97(5 \mathrm{H}, \mathrm{m}), 3.94-3.84(3 \mathrm{H}, \mathrm{m}), 3.67-3.56(4 \mathrm{H}, \mathrm{m})$, $3.34(3 \mathrm{H}, \mathrm{s}), 3.11(1 \mathrm{H}, \mathrm{d}, J 7.4 \mathrm{~Hz}), 3.07(1 \mathrm{H}, \mathrm{d}, J 7.3 \mathrm{~Hz})$, $2.47-2.43(3 \mathrm{H}, \mathrm{m}), 2.41-2.3(7 \mathrm{H}$, including t at 2.38 with $J 7.5 \mathrm{~Hz}), 1.63-1.10(292 \mathrm{H}, \mathrm{m}), 1.00(6 \mathrm{H}, \mathrm{d}, J 6.9 \mathrm{~Hz}), 0.84$ $(12 \mathrm{H}, \mathrm{t}, J 6.7 \mathrm{~Hz}), 0.65$

-0.56 (4H, m), $0.51(2 \mathrm{H}, \mathrm{dt}, J 7.6,4.0 \mathrm{~Hz}),-0.38$ (2H, br. q, $J$ $5.1 \mathrm{~Hz}) ; \delta_{\mathrm{C}}\left(101 \mathrm{MHz}, \mathrm{CDCl}_{3}+\right.$ few drops $\left.\mathrm{CD}_{3} \mathrm{OD}\right): 216.3$, 175.1, 175.0, 109.1, 107.9, 107.3, 83.0, 82.3, 81.8, 81.7, 81.0, $80.9,79.7,77.9,72.4,72.3,65.97,63.1,60.4,54.7,52.7,46.2$, 41.0, 34.7, 32.9, 31.7, 30.0, 29.5, 29.3, 29.2, 29.1, 28.5, 27.3, $27.1,25.5,23.5,22.5,16.1,15.6,13.8,10.7$; $v_{\max }: 3393$, $2919,2850,1713,1467,1100,720 \mathrm{~cm}^{-1}$. 


\section{Acknowledgments}

MM wishes to thank the Ministry of Higher Education \& Scientific Research - Kurdistan Region, Iraq for the award of a PhD grant.

\section{References and notes}

[1] M. Gengenbacher and S. H. E. Kaufmann, FEMS Microbiol. Rev., 2012, 36, 514-532.

[2] M. S. Glickman, W. R. Jacobs, N. York, and M. P. Ave, Cell, 2001, 104, $477-485$.

[3] World Health Organization (WHO; 2010) Tuberculosis. Media Centre: Factsheet No. 104.

[4] P. J. Brennan and H. Nikaido, Annu. Rev. Biochem., 1995, 64, 29-63.

[5] S. Sanchez, T. Bamhaoud, and J. Prandi, Eur. J. Org. Chem., 2002, 2002, 3864-3873.

[6] M. Miyauchi, M. Murata, K. Shibuya, E. Koga-Yamakawa, Y. Uenishi, N. Kusunose, M. Sunagawa, I. Yano, and Y. Kashiwazaki, Drug Discov Ther, 2011, 5, 130-135.

[7] D. E. Minnikin, L. Kremer, L. G. Dover, and G. S. Besra, Chem. Biol., $2002,9,545-553$.

[8] R. J. Anderson, R. E. Reeves, and F. H. Stodola, J.Biol.Chem., 1937, 121, $649-668$.

[9] I. Azuma, Y. Yamamura, and A. Misaki, J. Bacteriol., 1969, 98, 331333.

[10] M. McNeil, M. Daffe, and P. J. Brennan, J. Biol. Chem., 1991, 266, 13217-13223.

[11] G. S. Besra, K. H. Khoo, M. R. McNeil, a Dell, H. R. Morris, and P. J. Brennan, Biochemistry, 1995, 34, 4257-4266.

[12] J. D. Ayers, T. L. Lowary, C. B. Morehouse, and G. S. Besra, Bioorg. Med. Chem. Lett., 1998, 8, 437-442.

[13] H. B. Mereyala, S. Hotha, and M. K. Gurjar, Chem. Commun., 1998, 685-686.

[14] A. Ishiwata, H. Akao, Y. Ito, M. Sunagawa, N. Kusunose, and Y. Kashiwazaki, Bioorg. Med. Chem., 2006, 14, 3049-3061. [15] Y. Uenishi, N. Kusunose, I. Yano, and M. Sunagawa, $J$. Microbiol. Methods, 2010, 80, 302-305.

[16] C. Liu, M. R. Richards, and T. L. Lowary, J. Org. Chem., 2010, 75, 4992-5007.

[17] D. E. Minnikin, Biol. Mycobact., 1982, 1, 95-184.

[18] S. A. Cantrell, Mycobact. Dis., 2012, 02.

[19] V. Puech, M. Chami, A. Lemassu, M. A. Lanéelle, B. Schiffler, P. Gounon, N. Bayan, R. Benz, and M. Daffé, Microbiology, 2001, 147, 1365-1382.

[20] M. Goodfellow, The family Nocardiaceae, p. 1188-1213, A. Balows, H. G. Triiper, M. Dworkin, W. Harder, and K. H. Schleifer (ed.), Springer, Verlag, New York, 2nd ed., 1992, vol. 2.

[21] M. Watanabe, Y. Aoyagi, M. Ridell, and D. E. Minnikin, Microbiology, 2001, 147, 1825-1837.

[22] X.-Y. Liang, L.-M. Deng, X. Liu, and J.-S. Yang, Tetrahedron, 2010, 66, 87-93.

[23] F. W. D'Souza, J. D. Ayers, P. R. McCarren, and T. L. Lowary, J. Am. Chem. Soc., 2000, 122, 1251-1260.

[24] A. K. Sanki, J. Boucau, P. Srivastava, S. S. Adams, D. R. Ronning, and S. J. Sucheck, Bioorg. Med. Chem., 2008, 16, 5672-5682.

[25] I. A. Mikhailopulo and G. G. Sivets, Helv. Chim. Acta, 1999, 82, 2052-2065.

[26] A. N. Cuzzupe, R. Di Florio, and M. A. Rizzacasa, J. Org. Chem., 2002, 67, 4392-4398.

[27] J. R. Al Dulayymi, M. S. Baird, and E. Roberts, Tetrahedron, 2005, 61, 11939-11951.

[28] J. R. Al Dulayymi, M. S. Baird, E. Roberts, M. Deysel, J. Verschoor, Tetrahedron, 2007, 63, 2571-2592.

[29] G. Koza, C. Theunissen, J. R. Al Dulayymi, and M. S. Baird, Tetrahedron, 2009, 65, 10214-10229.

[30] M. O. Mohammed, M. S. Baird, J. R. Al Dulayymi, A. Jones, C. D. Gwenin, Tetrahedron, 2016, 72, 2849-2857.

[31] H. G. Tima, K. Huygen, and M. Romano, Expert Rev. Vaccines, 2016, $1-12$.
[32] H. G. Tima, J. R. Al Dulayymi, O. Denis, P. Lehebel, K. S. Baols, M. O. Mohammed, L. L'Homme, M. M. Sahb, G. Potemberg, S. Legrand, R. Lang, R. Beyaert, J. Piette, M. S. Baird, K. Huygen, M. Romano, J. Innate Immun., in press. [33] K. S. Baols, Ph.D. Thesis, Bangor University, 2014. 